Bull. Korean Math. Soc. 47 (2010), No. 4, pp. 793-803

DOI 10.4134/BKMS.2010.47.4.793

\title{
SOME WEAK HYPONORMAL CLASSES OF WEIGHTED COMPOSITION OPERATORS
}

\author{
Mohammad R. Jabbarzadeh and Mohammad R. Azimi
}

\begin{abstract}
In this note, we discuss measure theoretic characterizations for weighted composition operators in some operator classes on $L^{2}(\mathcal{F})$ such as, $p$-quasihyponormal, $p$-paranormal, $p$-hyponormal and weakly hyponormal. Some examples are then presented to illustrate that weighted composition operators lie between these classes.
\end{abstract}

\section{Introduction and preliminaries}

Let $\mathcal{H}$ be the infinite dimensional complex Hilbert space and let $\mathcal{L}(\mathcal{H})$ be the algebra of all bounded operators on $\mathcal{H}$. Let $A=U|A|$ be the canonical polar decomposition for $\mathrm{A} \in \mathcal{L}(\mathcal{H})$ and let $p \in(0, \infty)$. An operator $A$ is $p$-hyponormal if $\left(A^{*} A\right)^{p} \geq\left(A A^{*}\right)^{p}$ and $\mathrm{A}$ is $p$-quasihyponormal if $A^{*}\left(A^{*} A\right)^{p} A \geq A^{*}\left(A A^{*}\right)^{p} A$. For all unit vectors $x \in \mathcal{H}$, if $\left\||A|^{p} U|A|^{p} x\right\| \geq\left\||A|^{p} x\right\|^{2}$, then $A$ is called a $p$ paranormal operator. By using the property of real quadratic forms (see [10]), $A$ is $p$-paranormal if and only if

$$
|A|^{p} U^{*}|A|^{2 p} U|A|^{p}-2 k|A|^{2 p}+k^{2} \geq 0 \text { for all } k \geq 0 .
$$

An operator $A$ is normaloid if $\|A\|^{n}=\left\|A^{n}\right\|$ for all $n \in \mathbb{N}$. Let $\tilde{A}:=|A|^{\frac{1}{2}} U|A|^{\frac{1}{2}}$ be the Aluthge transform of $A$. An operator $A$ is defined to be weakly hyponormal if $|\tilde{A}| \geq|A| \geq\left|(\tilde{A})^{*}\right|$ (see [2]). There are several well-known relationships among these weaker than hyponormal classes (see [5]). The hierarchical relationship between the classes is as follows: $p$-hyponormal $\Rightarrow p$-quasihyponormal $\Rightarrow$ normaloid.

Let $(X, \mathcal{F}, \mu)$ be a complete $\sigma$-finite measure space and suppose that $T$ is a measurable transformation (i.e., $T^{-1} \mathcal{F} \subset \mathcal{F}$ ) from $X$ into $X$ such that $\mu \circ T^{-1}$ is absolutely continuous with respect to $\mu$, that is, $T$ is non-singular. Let $h$ be the Radon-Nikodym derivative $d \mu \circ T^{-1} / d \mu$ and we always assume that $h$ is almost everywhere finite-valued or, equivalently $\mathcal{A}:=T^{-1} \mathcal{F} \subseteq \mathcal{F}$ is a sub-sigma finite algebra, and we let $h_{n}:=d \mu \circ T^{-n} / d \mu$. The support of a measurable

Received February 25, 2009; Revised July 21, 2009.

2000 Mathematics Subject Classification. 47B20, $46 \mathrm{~B} 38$.

Key words and phrases. weighted composition operator, conditional expectation, $p$ paranormal, $p$-hyponormal, weakly hyponormal. 
function $f$ is defined by $\sigma(f)=\{x \in X: f(x) \neq 0\}$. All comparisons between two functions or two sets are to be interpreted as holding up to a $\mu$-null set.

For any non-negative $\mathcal{F}$-measurable functions $f$ as well as for any $f \in L^{p}(\mathcal{F})$, by the Radon-Nikodym theorem, there exists a unique $\mathcal{A}$-measurable function $E(f)$ such that

$$
\int_{B} E f d \mu=\int_{B} f d \mu \quad \text { for all } B \in \mathcal{A} .
$$

Hence we obtain an operator $E$ from $L^{2}(\mathcal{F})$ onto $L^{2}(\mathcal{A})$ which is called conditional expectation operator associated with the sub-sigma finite algebra $\mathcal{A}$. As an operator on $L^{2}(\mathcal{F}), E(\cdot)$ is the contractive orthogonal projection onto $L^{2}(\mathcal{A})$. It is easy to show that for each non-negative $\mathcal{F}$-measurable function $f$ or for each $f \in L^{2}(\mathcal{F})$, there exists a $\mathcal{F}$-measurable function $g$ such that $E(f)=g \circ T$. We can assume that $\sigma(g) \subseteq \sigma(h)$ and there exists only one $g$ with this property. We then write $g=E(f) \circ T^{-1}$ though we make no assumptions regarding the invertibility of $T$. For more details see $[7,8]$.

For a non-negative finite-valued $\mathcal{F}$-measurable function $u$, the weighted composition operator $W$ on $L^{2}(\mathcal{F})$ induced by $T$ and $u$ is given by

$$
W f:=\left(u C_{T}\right) f=u f \circ T, \quad f \in L^{2}(\mathcal{F}),
$$

where $C_{T}$ is the composition operator on $L^{2}(\mathcal{F})$ is defined by $C_{T} f=f \circ$ $T$. Here, the non-singularity of $T$ guarantees that $W$ is well defined as a mapping of equivalence classes of functions on $\sigma(u)$. Boundedness of weighted composition operators on $L^{p}(\mathcal{F})$ spaces already being studied in [7]. Namely, $W$ is bounded on $L^{p}(\mathcal{F})$ for $1 \leq p<\infty$ if and only if $J:=h E\left(|u|^{p}\right) \circ T^{-1} \in L^{\infty}(\mathcal{F})$. Throughout this paper we assume that $J \in L^{\infty}(\mathcal{F})$. The properties of this operators are studied by Harrington and Whitley [6], Lambert [7, 8], Singh and Manhas [9] and many other mathematicians.

The goal of this paper is to distinguish some weak hyponormal classes of weighted composition operators. Some results of the next section are generalizations of the work done in [3] and [4]. In those work Charles Burnap, Il Bong Jung and Alan Lambert determined when measure theoretic composition operators were $p$-hyponormal, $w$-hyponormal and other classes that are weaker than $p$-hyponormal. In Section 3, some examples are presented which show that weighted composition operators distinguish between these classes.

\section{Characterizations}

The following lemma is significant for amount of consideration for the next results and computations.

Lemma 2.1. Let $f \in L^{2}(\mathcal{F})$ and $A f:=u(h \circ T) E(u f)$. Then for all $p \in(0, \infty)$

$$
A^{p} f=u\left(h^{p} \circ T\right)\left[E\left(u^{2}\right)\right]^{p-1} E(u f) .
$$


Proof. Suppose $f \in L^{2}(\mathcal{F})$, then by induction we obtain

$$
A^{\frac{1}{n}} f=u \sqrt[n]{\frac{h \circ T}{\left[E\left(u^{2}\right)\right]^{n-1}}} E(u f), \quad n \in \mathbb{N} .
$$

Now the reiteration of powers of operator $A^{\frac{1}{n}}$, yields

$$
A^{\frac{m}{n}} f=u\left(\frac{h \circ T}{\left[E\left(u^{2}\right)\right]^{n-1}}\right)^{\frac{m}{n}}\left[E\left(u^{2}\right)\right]^{m-1} E(u f), \quad n \in \mathbb{N}, m \in \mathbb{Z} .
$$

Finally, by using of the functional calculus the desired formula is proved.

The function which plays the role for $W$, analogous to that which $h$ plays for $C_{T}$, is $J$. According to Theorem 2.3 in [3], one might conjecture that a generalization to the weighted case would say: $W$ is $p$-quasihyponormal if and only if $E\left(J^{p}\right) \geq J^{p} \circ T$. But, this fails to hold if $u$ is not $\mathcal{A}$-measurable.

In what follows, since for each $p>0$ and $f \geq 0$ a.e., $\sigma(f) \subseteq \sigma\left(E\left(f^{p}\right)\right)$, we use the notational convention of $\frac{u}{E\left(u^{2}\right)}$ for $\frac{u}{E\left(u^{2}\right)} \chi_{\sigma(u)}$ (see [4]).

Theorem 2.2. Let $W$ be a weighted composition operator on $L^{2}(\mathcal{F})$. Then the following statements are equivalent:

(i) $W$ is p-quasihyponormal.

(ii) $E\left(u^{2} J^{p}\right) \geq h^{p} \circ T\left[E\left(u^{2}\right)\right]^{p+1}$.

(iii) $W$ is p-paranormal.

Proof. (i) $\Leftrightarrow\left(\right.$ ii). Let $f \in L^{2}(\mathcal{F})$. By Lemma 2.1, it is easy to verify that

$$
W^{*}\left(W W^{*}\right)^{p} W f=h^{p+1}\left[E\left(u^{2}\right)\right]^{p+1} \circ T^{-1} f .
$$

Since $\left(W^{*} W\right)^{p} f=h^{p}\left[E\left(u^{2}\right)\right]^{p} \circ T^{-1} f=J^{p} f$, then we get that

$$
W^{*}\left(W^{*} W\right)^{p} W f=h\left[E\left(u^{2} J^{p}\right)\right] \circ T^{-1} f .
$$

Therefore, $W^{*}\left(W^{*} W\right)^{p} W \geq W^{*}\left(W W^{*}\right)^{p} W$ if and only if

$$
h\left[E\left(u^{2} J^{p}\right)\right] \circ T^{-1} \geq h^{p+1}\left[E\left(u^{2}\right)\right]^{p+1} \circ T^{-1} .
$$

Now composing with $T$ and using the fact that $h \circ T>0$, this is equivalent to $E\left(u^{2} J^{p}\right) \geq h^{p} \circ T\left[E\left(u^{2}\right)\right]^{p+1}$.

(ii) $\Leftrightarrow($ iii). Notice that the parts of the polar decomposition $U,|W|$ for $W$ are given by

$$
|W| f=\sqrt{J} f, \quad U f=\frac{u \cdot f \circ T}{\sqrt{h \circ T E\left(u^{2}\right)}}
$$

for all $f \in L^{2}(\mathcal{F})$. By a direct computation we have

$$
U^{*} f=h^{\frac{1}{2}}\left[\left[E\left(u^{2}\right)\right]^{-\frac{1}{2}} E(u f)\right] \circ T^{-1}
$$

and

$$
|W|^{p} U^{*}|W|^{2 p} U|W|^{p} f=h^{p}\left[E\left(u^{2}\right)\right]^{p-1} E\left(u^{2} J^{p}\right) \circ T^{-1} f .
$$

By the condition $(*), W$ is $p$-paranormal if and only if

$$
h^{p}\left[E\left(u^{2}\right)^{p-1} E\left(u^{2} J^{p}\right)\right] \circ T^{-1}-2 k h^{p}\left[E\left(u^{2}\right)^{p}\right] \circ T^{-1}+k^{2} \geq 0
$$




$$
\begin{aligned}
& \Leftrightarrow h^{p}\left[\left[E\left(u^{2}\right)\right]^{p-1} E\left(u^{2} J^{p}\right)\right] \circ T^{-1} \geq h^{2 p}\left[E\left(u^{2}\right)^{2 p}\right] \circ T^{-1} \\
& \Leftrightarrow E\left(u^{2} J^{p}\right) \geq h^{p} \circ T\left[E\left(u^{2}\right)\right]^{p+1} .
\end{aligned}
$$

Thus the theorem is proved.

To avoid tedious calculations the following theorem is stated only for composition operators.

Theorem 2.3. For a composition operator $C_{T}$ on $L^{2}(\mathcal{F})$, the following assertions hold.

(i) $C_{T}^{*}$ is p-quasihyponormal if and only if $h^{p} \circ T E(h) \geq h^{p+1}$.

(ii) $C_{T}^{*}$ is p-paranormal if and only if $h^{p} \circ T E\left(h^{\frac{p+1}{2}}\right) \geq h^{\frac{3 p+1}{2}}$.

Proof. (i) It is well known that, for each $f \in L^{2}(\mathcal{F})$,

$$
C_{T}^{*} f=h E(f) \circ T^{-1}, C_{T}^{*} C_{T} f=h f, C_{T} C_{T}^{*} f=h \circ T E(f) .
$$

Also, by Lemma 2.1 we have $\left(C_{T} C_{T}^{*}\right)^{p} f=(h \circ T)^{p} E(f)$,

$$
C_{T}\left(C_{T} C_{T}^{*}\right)^{p} C_{T}^{*} f=h^{p} \circ T^{2} E\left(h E(f) \circ T^{-1}\right) \circ T
$$

and

$$
C_{T}\left(C_{T}^{*} C_{T}\right)^{p} C_{T}^{*} f=h^{p+1} \circ T E(f) .
$$

Thus $C_{T}^{*}$ is $p$-quasihyponormal if and only if

$$
0 \leq\left\langle\left(C_{T}\left(C_{T} C_{T}^{*}\right)^{p} C_{T}^{*}-C_{T}\left(C_{T}^{*} C_{T}\right)^{p} C_{T}^{*}\right) f, f\right\rangle .
$$

Since $(X, \mathcal{A}, \mu)$ is a $\sigma$-finite measure space, let $f:=\chi_{T^{-1} B}$ with $\mu\left(T^{-1} B\right)<\infty$. Hence, the above inner product is non-negative if and only if

$$
0 \leq \int_{T^{-1} B}\left(h^{p} \circ T^{2} E\left(h E\left(\chi_{T^{-1} B}\right) \circ T^{-1}\right) \circ T-h^{p+1} \circ T E\left(\chi_{T^{-1} B}\right)\right) d \mu .
$$

Since $E\left(\chi_{T^{-1} B}\right) \circ T^{-1}=E\left(\chi_{B} \circ T\right) \circ T^{-1}=\chi_{B}$ on $\sigma(h)$, by change of variable theorem the previous integral is non-negative if and only if

$$
0 \leq \int_{B}\left(h^{p} \circ T E\left(h \chi_{B}\right)-h^{p+1} \chi_{B}\right) h d \mu=\int_{B}\left(h^{p} \circ T E(h)-h^{p+1}\right) h d \mu .
$$

But this is equivalent to $h^{p} \circ T E(h) \geq h^{p+1}$.

(ii) Let $f \in L^{2}(\mathcal{F})$. The partial isometry operator and its adjoint in the polar decomposition of $C_{T}^{*}$ are

$$
U f=h^{\frac{1}{2}} E(f) \circ T^{-1} \quad \text { and } \quad U^{*} f=(h \circ T)^{-\frac{1}{2}} f \circ T .
$$

Therefore, by Lemma 2.1

$$
\left|C_{T}^{*}\right|^{p} U^{*}\left|C_{T}^{*}\right|^{2 p} U\left|C_{T}^{*}\right|^{p} f=h^{\frac{p-1}{2}} \circ T h^{p} \circ T^{2} E\left(h^{\frac{p+1}{2}} E(f) \circ T^{-1}\right) \circ T .
$$

Now, by the condition $(*), C_{T}^{*}$ is $p$-paranormal if and only if

$$
\left\langle h^{\frac{p-1}{2}} \circ T h^{p} \circ T^{2} E\left(h^{\frac{p+1}{2}} E(f) \circ T^{-1}\right) \circ T-2 h^{p} \circ T E(f) k+k^{2}, f\right\rangle \geq 0 .
$$


Put $f:=\chi_{T^{-1} B}$ with $\mu\left(T^{-1} B\right)<\infty$. Hence the above inner product is nonnegative if and only if

$$
\begin{gathered}
\int_{\chi_{T^{-1} B}}\left(h^{\frac{p-1}{2}} \circ T h^{p} \circ T^{2} E\left(h^{\frac{p+1}{2}} E\left(\chi_{T^{-1} B}\right) \circ T^{-1}\right) \circ T-2 h^{p} \circ T E\left(\chi_{T^{-1} B}\right) k+k^{2}\right) d \mu \\
=\int_{B}\left(h^{\frac{p-1}{2}} h^{p} \circ T E\left(h^{\frac{p+1}{2}} \chi_{B}\right)-2 h^{p} \chi_{B} k+k^{2}\right) h d \mu \geq 0 .
\end{gathered}
$$

But this is possible if and only if $h^{2 p}-h^{\frac{p-1}{2}} h^{p} \circ T E\left(h^{\frac{p+1}{2}}\right) \leq 0$, since $h$ is a non-negative function in $L^{2}(\mathcal{F})$ and $B$ is an arbitrary element of sigma finite algebra $\mathcal{F}$. So the proof of (ii) is therefore complete.

Corollary 2.4. Let $h \in L^{\infty}(\mathcal{A})$. The followings are equivalent.

(i) $C_{T}^{*}$ is p-quasihyponormal.

(ii) $h \circ T \geq h$ on $\sigma(h)$.

(iii) $C_{T}^{*}$ is p-paranormal.

Proof. Since $h \in L^{\infty}(\mathcal{A})$, then $E(h)=h$ and $E\left(h^{\frac{p+1}{2}}\right)=h^{\frac{p+1}{2}}$. Now the rest is obvious by Theorem 2.3 .

Lemma 2.5. For every $f \in L^{2}(\mathcal{F})$,

$$
\int_{X} \alpha|f|^{2} d \mu \geq \int_{X}|E(\beta f)|^{2} d \mu
$$

if and only if $\sigma(\beta) \subset \sigma(\alpha)$ and $E\left(\frac{\beta^{2}}{\alpha} \chi_{\sigma(\alpha)}\right) \leq 1$.

Proof. See [4] and [8].

The following theorem is a generalization of Theorem 2.4 in [4].

Theorem 2.6. $W$ is p-hyponormal if and only if $\sigma(u) \subseteq \sigma(J)$ and

$$
h^{p} \circ T E\left(\frac{u^{2}\left(E\left(u^{2}\right)\right)^{p-1} \chi_{\sigma(J)}}{J^{p}}\right) \leq 1 .
$$

Proof. First notice that for every $f \in L^{2}(\mathcal{F})$,

$$
\left\langle\left(W^{*} W\right)^{p} f, f\right\rangle=\int_{X} h^{p}\left[E\left(u^{2}\right)\right]^{p} \circ T^{-1}|f|^{2} d \mu
$$

and

$$
\begin{aligned}
\left\langle\left(W W^{*}\right)^{p} f, f\right\rangle & =\int_{X} u h^{p} \circ T\left[E\left(u^{2}\right)\right]^{p-1} E(u f) \bar{f} d \mu \\
& =\int_{X}\left|E\left(h^{\frac{p}{2}} \circ T\left[E\left(u^{2}\right)\right]^{\frac{p-1}{2}} u f\right)\right|^{2} d \mu .
\end{aligned}
$$

Hence by Lemma 2.5, $W$ is $p$-hyponormal if and only if

$$
\sigma\left(\left(u h^{\frac{p}{2}} \circ T\left(E\left(u^{2}\right)\right)^{\frac{p-1}{2}}\right)\right) \subseteq \sigma(J)
$$


and

$$
E\left(\frac{h^{p} \circ T u^{2}\left(E\left(u^{2}\right)\right)^{p-1}}{h^{p}\left(E\left(u^{2}\right)\right)^{p} \circ T^{-1}} \chi_{\sigma(J)}\right) \leq 1 .
$$

But these are then equivalent to $\sigma(u)=\sigma\left(u E\left(u^{2}\right)^{\frac{p-1}{2}}\right) \subseteq \sigma(J)$ and

$$
h^{p} \circ T E\left(\frac{u^{2}\left(E\left(u^{2}\right)\right)^{p-1} \chi_{\sigma(J)}}{J^{p}}\right) \leq 1 .
$$

Recall that the Aluthge transform of operator $A \in \mathcal{L}(\mathcal{H})$ is the operator $\tilde{A}$ given by $\tilde{A}:=|A|^{\frac{1}{2}} U|A|^{\frac{1}{2}}$. For a such operator $A \in \mathcal{L}(\mathcal{H})$ and $0<r \leq 1$, put $A_{r}:=|A|^{r} U|A|^{1-r}$ (see [1]). Then $A_{\frac{1}{2}}$ is exactly the Aluthge transform of operator $A$. Here, the following lemma describes $A_{r},\left|A_{r}\right|$ and $\left|A_{r}^{*}\right|$ of a weighted composition operator by using conditional expectation operator.

Lemma 2.7. For a weighted composition operator $W$ we have the following entities

$$
W_{r} f=\omega_{r} \cdot f \circ T, \quad\left|W_{r}\right| f=\sqrt{h\left[E\left(\omega_{r}^{2}\right)\right] \circ T^{-1}} f
$$

and

$$
\left|W_{r}^{*}\right| f=P_{v_{r}} f:=v_{r} E\left(v_{r} f\right),
$$

where $\omega_{r}:=u\left(\frac{J \chi_{\sigma(E(u))}}{h \circ T E\left(u^{2}\right)}\right)^{\frac{r}{2}}$ and $v_{r}:=\frac{\omega_{r} \sqrt{h \circ T}}{\sqrt[4]{E\left(\left[\omega_{r} \sqrt{h \circ T}\right]^{2}\right)}}$.

Proof. Since $\sigma\left(v_{r}\right)=\sigma\left(\omega_{r}\right)=\sigma(J) \cap \sigma(u)$, one may verify that the mentioned results hold.

The purpose of the next theorem is to characterize weakly hyponormal weighted composition operators which is similar to Theorem 2.8 in [4].

Theorem 2.8. Let $W$ be a weighted composition operators on $L^{2}(\mathcal{F})$. Then

(i) $\left|W_{r}\right| \geq|W|$ if and only if $E\left(\omega_{r}^{2}\right) \geq E\left(u^{2}\right)$.

(ii) $|W| \geq\left|W_{r}^{*}\right|$ if and only if $E\left(\frac{v_{r}^{2}}{\sqrt{J}} \chi_{\sigma(J)}\right) \leq 1$.

Proof. (i) It is trivial.

(ii) As for this assertion, since $\left|W_{r}^{*}\right|=P_{v_{r}}$ we have,

$$
\begin{aligned}
|W| \geq\left|W_{r}^{*}\right| & \Leftrightarrow \forall f \in L^{2}(\mathcal{F}), \quad\left\langle\left(W^{*} W\right)^{\frac{1}{2}} f, f\right\rangle \geq\left\langle\left|W_{r}^{*}\right| f, f\right\rangle \\
& \Leftrightarrow \int_{X} \sqrt{J}|f|^{2} d \mu \geq \int_{X} v_{r} E\left(v_{r} f\right) \bar{f} d \mu=\int_{X}\left|E\left(v_{r} f\right)\right|^{2} d \mu .
\end{aligned}
$$

Since $\sigma\left(\omega_{r}\right) \subseteq \sigma(J)$, then by Lemma 2.5, $|W| \geq\left|W_{r}^{*}\right|$ if and only if $\sigma\left(\omega_{r}\right) \subset$ $\sigma(J)$ and $E\left(\frac{v_{r}^{2}}{\sqrt{J}} \chi_{\sigma(J)}\right) \leq 1$.

Now, we are going to investigate when the weighted composition operator is normaloid. Suppose that $W$ is a bounded weighted composition operator on $L^{2}(\mathcal{F})$. We define the measure $\mu_{u^{2}, T^{n}}$ by

$$
\mu_{u^{2}, T^{n}}(F)=\int_{T^{-n}(F)}|u|^{2} d \mu, \quad n \in \mathbb{N}, F \in \mathcal{F} .
$$


The assumption $\mu \circ T^{-1} \ll \mu$ implies that $\mu_{u^{2}, T^{n}} \ll \mu$. Consequently, there exists the Radon-Nikodym derivative $H_{n}:=\frac{d \mu_{u^{2}, T^{n}}}{d \mu}$. Also we have a chain

$$
\mu_{u^{2}, T^{n}} \ll \mu \circ T^{-n} \ll \cdots \ll \mu \circ T^{-2} \ll \mu \circ T^{-1} \ll \mu .
$$

Proposition 2.9. Let $W$ be a weighted composition operator on $L^{2}(\mathcal{F})$. Then $\left\|W^{n}\right\|=\left\|M_{\sqrt{H_{n}}}\right\|$ for all $n \in \mathbb{N}$, where $M_{\alpha}$ means a multiplication operator, i.e., $M_{\alpha} f=\alpha f$.

Proof. Let $f \in L^{2}(\mathcal{F})$, by calculating the $n$th iteration of $W$ we will have

$$
W^{n} f=\prod_{i=0}^{n-1}\left(u \circ T^{i}\right)\left(f \circ T^{n}\right) .
$$

Thus,

$$
\begin{aligned}
\left\|W^{n} f\right\|^{2}= & \int_{X}\left|\prod_{i=0}^{n-1}\left(u \circ T^{i}\right)\left(f \circ T^{n}\right)\right|^{2} d \mu \\
= & \int_{X} \prod_{i=0}^{n-2}\left|u \circ T^{i}\right|^{2}\left|f \circ T^{n-1}\right|^{2} d \mu_{u^{2}, T} \\
= & \int_{X} \prod_{i=0}^{n-3}\left|u \circ T^{i}\right|^{2}\left|f \circ T^{n-2}\right|^{2} d \mu_{u^{2}, T^{2}} \\
& \vdots \\
= & \int_{X}|f|^{2} d \mu_{u^{2}, T^{n}}=\int_{X} H_{n}|f|^{2} d \mu \\
= & \int_{X}\left|M_{\sqrt{H_{n}}} f\right|^{2} d \mu=\left\|M_{\sqrt{H_{n}}} f\right\|^{2} .
\end{aligned}
$$

Thus the proposition is proved.

Remark 2.10. Put $E_{n}:=E\left(\cdot \mid T^{-n} \mathcal{F}\right)$. Since $d \mu_{u^{2}, T^{n}}=h_{n}\left[E_{n}\left(|u|^{2}\right)\right] \circ T^{-n} d \mu$, it follows that $H_{n}=h_{n}\left[E_{n}\left(|u|^{2}\right)\right] \circ T^{-n}$. Also, since $\|W\|=\|J\|_{\infty}^{\frac{1}{2}}$ and $\left\|W^{n}\right\|=$ $\left\|\sqrt{H_{n}}\right\|_{\infty}$ (Proposition 2.9), $W$ is normaloid if and only if $\|J\|_{\infty}=\left\|H_{n}\right\|_{\infty}^{\frac{1}{n}}$ for all $n \in \mathbb{N}$.

\section{Examples}

Example 3.1. Let $w=\left\{m_{n}\right\}_{n=1}^{\infty}$ be a sequence of positive real numbers. Consider the space $l^{p}(w)=L^{p}\left(\mathbb{N}, 2^{\mathbb{N}}, \mu\right)$, where $2^{\mathbb{N}}$ is the power set of natural numbers and $\mu$ is a measure on $2^{\mathbb{N}}$ defined by $\mu(\{n\})=m_{n}$. Let $u=\left\{u_{n}\right\}_{n=1}^{\infty}$ be a sequence of non-negative real numbers. Let $T: \mathbb{N} \rightarrow \mathbb{N}$ be a non-singular 
measurable transformation; i.e., $\mu \circ T^{-1} \ll \mu$. Direct computation shows that

$$
h(k)=\frac{1}{m_{k}} \sum_{j \in T^{-1}(k)} m_{j}, \quad E(f)(k)=\frac{\sum_{j \in T^{-1}(T(k))} f_{j} m_{j}}{\sum_{j \in T^{-1}(T(k))} m_{j}}
$$

for all non-negative sequence $f=\left\{f_{n}\right\}_{n=1}^{\infty}$ and $k \in \mathbb{N}$.

By Theorem 2.2, $W$ is $p$-paranormal ( $p$-quasihyponormal) if and only if

$$
\sum_{j \in T^{-1}(T(k))}(u(j))^{2}(J(j))^{p} m_{j} \geq m_{T(k)}\left\{\frac{\sum_{j \in T^{-1}(T(k))}(u(j))^{2} m_{j}}{m_{T(k)}}\right\}^{p+1} .
$$

Also, by Theorem 2.3, $C_{T}^{*}$ is $p$-quasihyponormal if and only if

$$
\frac{1}{m_{T(k)}^{p}}\left\{\sum_{j \in T^{-1}(T(k))} m_{j}\right\}^{p-1}\left\{\sum_{j \in T^{-1}(T(k))} h(j) m_{j}\right\} \geq \frac{1}{m_{k}^{p+1}}\left\{\sum_{j \in T^{-1}(k)} m_{j}\right\}^{p+1}
$$

and $C_{T}^{*}$ is $p$-paranormal if and only if

$$
\frac{1}{m_{T(k)}^{p}}\left\{\sum_{j \in T^{-1}(T(k))} m_{j}\right\}^{p-1}\left\{\sum_{j \in T^{-1}(T(k))}(h(j))^{\frac{p+1}{2}} m_{j}\right\} \geq\left\{\frac{1}{m_{k}} \sum_{j \in T^{-1}(k)} m_{j}\right\}^{\frac{3 p+1}{2}} .
$$

Example 3.2. Let $X=[1, \infty)$, equipped with the Lebesgue measure $\mu$ on the Lebesgue measurable subsets. The transformation $T$ and the weight function $u(x)$ are given by $T(x)=\sqrt{x}$ and $u(x)=\frac{1}{\sqrt{1+x}}$. Then $h(x)=2 x, J=\frac{2 x}{1+x^{2}}$, $h \circ T(x)=2 \sqrt{x}, J \circ T(x)=\frac{2 \sqrt{x}}{1+x}, E=I$ (identity operator on $L^{2}(\mathcal{F})$ ) and $\sigma(J)=X$. In this case, by Theorems 2.2 and 2.6 the $p$-quasihyponormality, $p$-paranormality and $p$-hyponormality of $W$ is equivalent to $J \geq J \circ T$. Therefore $W$ does not lie in the above classes while $C_{T}$ is $p$-quasihyponormal, $p$ paranormal and also is $p$-hyponormal. Clearly, by Theorem $2.3 C_{T}^{*}$ is not $p$ quasihyponormal. But both of the operators $C_{T}$ and $\widetilde{C_{T}}$ are $p$-quasihyponormal, since $\widetilde{C_{T}}$ is a weighted composition operator with weight function $u=\left(\frac{h}{h \circ T}\right)^{\frac{1}{4}}$ and then follow according to Theorem 2.2 .

However, if we change only the underlying space to $X=(0,1)$, then by Theorem 2.3 for each $p>0, C_{T}^{*}$ is $p$-quasihyponormal while none of the operators $C_{T}$ and $\widetilde{C_{T}}$ are not $p$-quasihyponormal.

Example 3.3. Let $X$ be the set of nonnegative integers, let $\mathcal{F}$ be the $\sigma$-algebra of all subsets of $X$, and take $\mu$ to be the point mass measure determined by the

$$
m=1,1,1, c, d, c^{2}, d^{2}, c^{3}, d^{3}, \ldots,
$$

where $c$ and $d$ are fixed positive real numbers. Define

$$
T(k)=\left\{\begin{array}{lc}
0 & k=0,1,2 \\
k-2 & k \geq 3
\end{array}\right.
$$


Note that this interesting example was used in [3] and [4] to show that composition operators can separate almost all weak hyponormal classes. Define

$$
u(k)=\left\{\begin{array}{cc}
1 & k=0,1,2 \\
k & k \geq 3
\end{array}\right.
$$

Now, consider some useful computations as follows:

$$
\begin{gathered}
h_{n}(k)=\frac{1}{m_{k}} \sum_{j \in T^{-1}(k)} h_{n-1}(j) m_{j}, \\
J(k)=\frac{1}{m_{k}} \sum_{j \in T^{-1}(k)}(u(j))^{2} m_{j}, \quad H_{n}(k)=\frac{1}{m_{k}} \sum_{j \in T^{-n}(k)}(u(j))^{2} m_{j}, \\
\left(E\left(u^{2}\right) \circ T^{-1}\right)(k)=\frac{\sum_{j \in T^{-1}(k)}(u(j))^{2} m_{j}}{\sum_{j \in T^{-1}(k)} m_{j}} .
\end{gathered}
$$

It is easy to verify that

$$
\begin{aligned}
h & =3, c, d, c, d, \ldots, \\
h \circ T & =3,3,3, c, d, c, d, \ldots, \\
E\left(u^{2}\right) & =1,1,1,9,16,25, \ldots,
\end{aligned}
$$

and

$$
\begin{aligned}
& h_{2}: 3+(c+d), c^{2}, d^{2}, c^{2}, d^{2}, \ldots \\
& h_{3}: 3+(c+d)+\left(c^{2}+d^{2}\right), c^{3}, d^{3}, c^{3}, d^{3}, \ldots \\
& h_{n}: 1+\frac{c^{n}-1}{c-1}+\frac{d^{n}-1}{d-1}, c^{n}, d^{n}, c^{n}, d^{n}, \ldots
\end{aligned}
$$

Now fix a number $p>0$, then

$$
J^{p}(k)=\left\{\begin{array}{lc}
3^{p} & k=0 \\
(2 n+2)^{2 p} d^{p} & k=2 n \\
(2 n+1)^{2 p} c^{p} & k=2 n-1,
\end{array}\right.
$$

and

$$
E\left(u^{2} J^{p}\right)(k)=\left\{\begin{array}{lc}
\frac{1}{3}\left(3^{p}+9^{p} c^{p}+16^{p} d^{p}\right) & k=0,1,2 \\
4 n^{2}(2 n+2)^{2 p} d^{p} & k=2 n \\
(2 n-1)^{2}(2 n+1)^{2 p} c^{p} & k=2 n-1 .
\end{array}\right.
$$

By Theorem 2.2, $W$ is $p$-paranormal if and only if

$$
3^{p} c^{p}+\left(\frac{16}{3}\right)^{p} d^{p} \geq 2
$$

and this inequality holds if $c \in(0.5, \infty), d \in(0.2, \infty)$. 
With the new weight function $u$ given by

$$
u(k)=\left\{\begin{array}{cc}
1 & k=0,1,2 \\
c & \text { for odd } k \geq 3 \\
d & \text { for even } k \geq 3
\end{array}\right.
$$

we have

$$
\begin{aligned}
J & : 3, c^{3}, d^{3}, c^{3}, d^{3}, \ldots \\
H_{1} & : 3, c^{3}, d^{3}, c^{3}, d^{3}, \ldots \\
H_{2} & : 3+\left(c^{3}+d^{3}\right), c^{4}, d^{4}, c^{4}, d^{4}, \ldots \\
H_{3} & : 3+\left(c^{3}+d^{3}\right)+\left(c^{4}+d^{4}\right), c^{5}, d^{5}, c^{5}, d^{5}, \ldots \\
\quad & \vdots \\
H_{n} & : 3+\frac{c^{n+2}-c^{3}}{c-1}+\frac{d^{n+2}-d^{3}}{d-1}, c^{n+2}, d^{n+2}, c^{n+2}, d^{n+2}, \ldots
\end{aligned}
$$

If $c<\sqrt[3]{3}$ and $d<\sqrt[3]{3}$, then $\|J\|_{\infty}=3$, since $\left\|H_{n}\right\|_{\infty}^{\frac{1}{n}}<3$, thus $W$ cannot be normaloid.

Example 3.4. Let $X=[0,1]$, equipped with the Lebesgue measure $\mu$ on the Lebesgue measurable subsets. The transformation $T: X \rightarrow X$ given by $T(x)=2 x(1-x)$. Direct computation shows that $h(x)=\frac{1}{2 \sqrt{1-2 x}} \chi_{\left[0, \frac{1}{2}\right)}(x)$ and for each $f \in L^{2}(\mathcal{F})$,

$$
E(f)(x)=\frac{1}{2}[f(x)+f(1-x)] \chi_{\left[0, \frac{1}{2}\right)}(x)
$$

and

$$
h E(f) \circ T^{-1}(x)=\frac{1}{2 \sqrt{1-2 x}}\left[f\left(\frac{1}{2}-\frac{1}{2} \sqrt{1-2 x}\right)+f\left(\frac{1}{2}+\frac{1}{2} \sqrt{1-2 x}\right)\right] \chi_{\left[0, \frac{1}{2}\right)}(x) .
$$

With given weight function $u(x)=x-\frac{1}{2}$, we have $J(x)=\frac{1}{4} \sqrt{1-2 x} \chi_{\left[0, \frac{1}{2}\right)}(x)$, $E\left(u^{2}\right)(x)=\left(x-\frac{1}{2}\right)^{2} \chi_{\left[0, \frac{1}{2}\right)}(x)$ and for a fix number $p>0$,

$$
E\left(u^{2} J^{p}\right)(x)=\frac{4^{-(p+1)}}{2}(1-2 x)^{\frac{p}{2}+2}\left[1+(-1)^{\frac{p}{2}+2}\right] \chi_{\left[0, \frac{1}{2}\right)}(x) .
$$

Now we confine our attention to a nonnegative integer $p$. If $\frac{p}{2}+2$ is an odd number, then $E\left(u^{2} J^{p}\right)=0$ on $\left[0, \frac{1}{2}\right)$, hence $W$ cannot be $p$-paranormal operator. However, if $\frac{p}{2}+2$ is an even number, then $W$ is $p$-paranormal.

Acknowledgment. The authors would like to express their deep gratitude to the referee(s) for his/her careful reading of the paper and helpful comments which improved the presentation of it. 


\section{References}

[1] A. Aluthge, On p-hyponormal operators for $0<p<1$, Integral Equations Operator Theory 13 (1990), no. 3, 307-315.

[2] A. Aluthge and D. Wang, w-hyponormal operators, Integral Equations Operator Theory 36 (2000), no. 1, 1-10.

[3] C. Burnap and I. Jung, Composition operators with weak hyponormality, J. Math. Anal. Appl. 337 (2008), no. 1, 686-694.

[4] C. Burnap, I. Jung, and A. Lambert, Separating partial normality classes with composition operators, J. Operator Theory 53 (2005), no. 2, 381-397.

[5] T. Furuta, Invitation to Linear Operators, Taylor \& Francis, Ltd., London, 2001.

[6] D. Harrington and R. Whitley, Seminormal composition operators, J. Operator Theory 11 (1984), no. 1, 125-135.

[7] T. Hoover, A. Lambert, and J. Quinn The Markov process determined by a weighted composition operator, Studia Math. 72 (1982), no. 3, 225-235.

[8] A. Lambert, Hyponormal composition operators, Bull. London Math. Soc. 18 (1986), no. 4, 395-400.

[9] R. K. Singh and J. S. Manhas, Composition Operators on Function Spaces, NorthHolland Mathematics Studies, 179. North-Holland Publishing Co., Amsterdam, 1993.

[10] T. Yamazaki and M. Yanagida, A further generalization of paranormal operators, Sci. Math. 3 (2000), no. 1, 23-31.

MOHAmmad R. JabBarzadeH

Faculty of Mathematical Sciences

UNIVERSITY OF TABRIZ

P. O. Box: 5166615648, TABRIZ, IRAN

E-mail address: mjabbar@tabrizu.ac.ir

Mohammad R. Azimi

Faculty of Mathematical Sciences

UNIVERSITY OF TABRIZ

P. O. Box: 5166615648, TABrIZ, IrAN

E-mail address: mh_azimi@tabrizu.ac.ir 